\title{
Outage Performance of CRNs with Best Relay Selection in Dependent Nonidentical Distributed Nakagami-m Channels
}

\author{
Zongsheng Zhang, ${ }^{1}$ Jinlong Wang, ${ }^{1}$ Qihui $\mathrm{Wu},{ }^{1}$ and Xurong $\mathrm{Pi}^{2}$ \\ ${ }^{1}$ College of Communications Engineering, PLA University of Science and Technology, Nanjing 21007, China \\ ${ }^{2}$ Department of Reconnaissance and Survey, Nanjing Artillery Academy, Nanjing 210013, China \\ Correspondence should be addressed to Zongsheng Zhang; zhangzongsheng1984@163.com
}

Received 22 November 2013; Accepted 3 March 2014; Published 13 April 2014

Academic Editor: Xin Wang

Copyright (c) 2014 Zongsheng Zhang et al. This is an open access article distributed under the Creative Commons Attribution License, which permits unrestricted use, distribution, and reproduction in any medium, provided the original work is properly cited.

The outage performance with best relay selection is proposed for cognitive relay networks with multiple primary users in independent nonidentical distributed Nakagami-m fading channels. Specifically, we take the interference temperature and fading severity into consideration. Exact closed-form expression of outage probability is derived. Based on the exact closed-form expression, we can evaluate the impact of interference temperature, fading severity, number of relays, and number of primary users on the secondary network. Finally, the effects of fading severity, number of relays, number of primary users, and interference temperature on the system performance are examined through some representative numerical plots, and the Monte Carlo results match perfectly with theory results which validates our theory analysis.

\section{Introduction}

The wireless communications have dramatically grown over the past decades and this trend is expected to continue with new and increasingly bandwidth-hungry mobile applications and services introduced every day. Meanwhile, according to the Federal Communications Commission (FCC), large portions of the licensed wireless spectrum resources are underutilized [1]. As such, cognitive radio (CR) has recently been evolving as a promising technology to attain much higher spectrum efficiency by dynamic access [2-5]. In CR networks, unlicensed users (secondary users) are allowed to reuse the licensed spectrum opportunistically if they do not cause harmful interference to the licensed users (primary users). As a result, this new communication technology can improve spectrum utilization dramatically.

Cooperative diversity [6], emerging as a new spatial diversity technique, can effectively combat channel fading and enhance the throughput. The advantages of such cooperative diversity protocols proposed in [7-9] come at the expense of a reduction in spectral efficiency since the cooperative relays will transmit on orthogonal channels. The inefficient utilization of the channel resources in regular cooperative networks can be enhanced by using the relay selection strategy [10], where only the best relay is selected to forward a source node's signal and thus only two channels (the best relay link and direct link) are required regardless of the number of relays.

Cooperation, in general, has great potential to be used in cognitive radio networks. By implementing a relay between base station and mobile node, the path loss can be reduced which leads to reduction of the outage probability as well as the enhancement of the coverage. The huge potential of achieving both spectrum sharing and relay benefits has resulted in enormous interest in cognitive relay networks [1114]. In [11], it was shown that introducing a relay in cognitive networks will improve outage performance relative to its direct link counterpart. Assuming the presence of a direct link in the cognitive system, a tight lower bound expression for the outage probability of cognitive relay networks was derived in [12]. In [13], the exact outage probability of underlay cognitive networks adopting decode-and-forward (DF) relaying considering best relay selection in Rayleigh fading channels has been derived. Most recently, the closed-form 
expression of outage probability in cognitive relay networks considering mutual interferences in multiple primary users has been derived in [14].

The work done in [11-14] was great and provided a good understanding on the outage performance of cognitive relay networks. However, most of them assumed Rayleigh fading environment. Recently, owing to this fact, some studies [15-18] considered the outage performance in Nakagami$\mathrm{m}$ fading environment. In [15], the exact outage probability of underlay cognitive relay networks using amplify-andforward (AF) relaying in Nakagami-m fading was derived. The outage probability of DF cognitive dual-hop systems was investigated, considering joint constraints on the peak and average interference powers at the primary receiver in [16]. In [17], the outage probability of dual-hop cognitive relay networks considering the direct link has been derived. Most recently, the outage performance of dual-hop cognitive relay networks was analyzed considering multiple PUs in Nakagami-m fading channels in [18].

However, the work done in [15-18] has just considered single relay, ignoring the benefits of diversity of relay selection. To the best of our knowledge, the outage performance of dual-hop cognitive relay networks considering relay selection with multiple primary users in Nakagami-m fading channels is almost unexplored from the analytical point of view. To fill this important gap, we will derive the closed-form outage probability expression in such an environment in this paper. The remaining of this paper is organized as follows. After introducing the system model and channel model in Section 2, the closed-form outage probability expression is derived in Section 3. In Section 4, numerical results are presented to validate the theory analysis. Finally, some conclusions are drawn in Section 5.

\section{System Model}

We consider underlay cognitive relay networks (in this paper, primary users are assumed closely located to be a cluster. As such, we assume that the distance between primary receivers is small compared to the distance between CT and each primary receiver. Therefore, for simplicity of analysis, the path loss is the same for CT and any primary receivers and is not taken into consideration in this paper) with the coexistence of primary network and secondary networks, as depicted in Figure 1. In the secondary networks, cognitive transmitter (CT) transmits its data to cognitive destination (CD) with the help of $M$ cognitive relays $(R)$. Notice that $M$ cognitive relays denoted by $R=\left\{R_{i} \mid i=1, \ldots, M\right\}$ are available to assist CT's data transmission and the DF protocol is considered throughout this paper. Moreover, we adopt the best relay selection scheme in this paper. Specifically, we denote $h_{1 i}, h_{2 i}, h_{3 j}$, and $h_{i j}$ as the independent, nonidentical distributed channel links for the CT- $R_{i}, R_{i}$-CD, CT- $P R_{j}$, and $R_{i}-P R_{j}$, respectively. These channel gains are modeled as Nakagami-m random variables. Then, the effective power channel gains $\left|h_{1 i}\right|^{2},\left|h_{2 i}\right|^{2},\left|h_{3 j}\right|^{2}$, and $\left|h_{i j}\right|^{2}$ follow the gamma distribution with different fading parameters $m_{1}, m_{2}, m_{3}$,

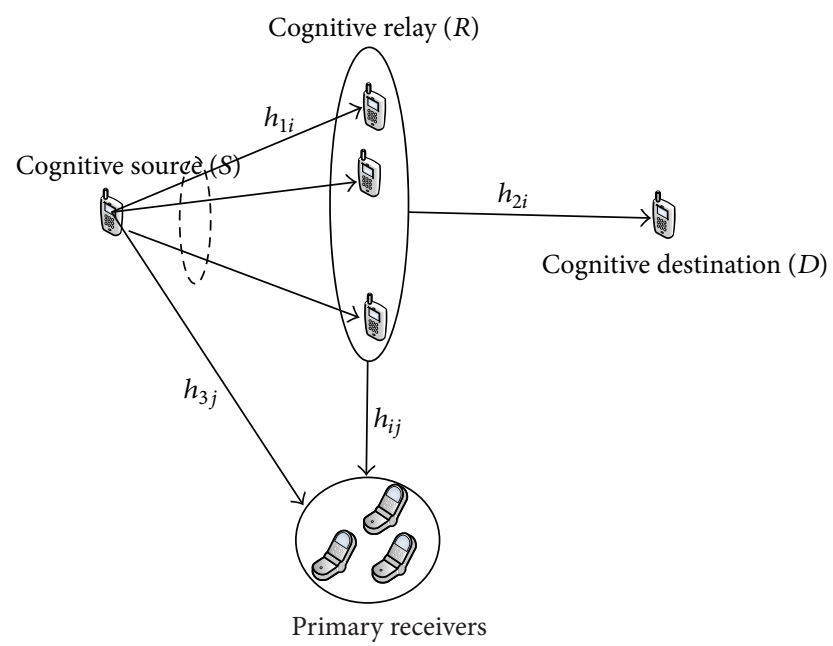

Figure 1: System model.

and $m_{4}$ and channel powers $\Omega_{1}, \Omega_{2}, \Omega_{3}$, and $\Omega_{4}$, respectively. Therefore, the probability density function (PDF) and cumulative distribution function (CDF) of $X$, for $X \in$ $\left\{\left|h_{1 i}\right|^{2},\left|h_{2 i}\right|^{2},\left|h_{3 j}\right|^{2},\left|h_{i j}\right|^{2}\right\}$, can be formulated as

$$
\begin{gathered}
f_{|h|^{2}}(x)=\frac{\beta^{m}}{\Gamma(m)} x^{m-1} e^{-\beta x}, \\
F_{|h|^{2}}(x)=\frac{\gamma(m, \beta x)}{\Gamma(m)},
\end{gathered}
$$

respectively, where $\beta=m / \Omega, \Gamma(\cdot)$ denotes the gamma function, and $\gamma(\cdot, \cdot)$ represents the incomplete gamma function [19].

The communications in the secondary system occur over a dual-hop transmission consisting of two distinct phases. In the first phase, the CT transmits its signal to all cognitive relays. In the second phase, the best relay in the decoding set forwards the signal to the cognitive destination. In order to avoid harmful interference to primary user, the secondary system should limit its transmit power. As such, the transmit powers at $\mathrm{CT}$ and $R$ can be mathematically written as

$$
\begin{aligned}
& P_{S}=\frac{Q}{\max _{j}\left|h_{3 j}\right|^{2}}, \\
& P_{R}=\frac{Q}{\max _{j}\left|h_{i j}\right|^{2}},
\end{aligned}
$$

where $Q$ denotes the maximum allowable interference power of primary receiver.

\section{Outage Probability Analysis}

This section focuses on the best relay selection problem in cognitive relay networks considering independent and nonidentical distributed Nakagami-m fading. As has been stated in Section 2, the adaptive cooperation scheme is divided into 
two phases. As such, in the first phase, the received signal at the candidate relay $R_{i}$ can be expressed as

$$
y_{R_{i}}=\sqrt{P_{S}} h_{1 i} x_{s}+n_{i},
$$

where $x_{s}$ denotes the signal transmitted by CT and $n_{i}$ represents the additive white Gaussian noise (AWGN) at the $R_{i}$ with zero mean and power spectral density $N_{0}$. For simplicity of analysis, we set $E\left[\left|x_{s}\right|^{2}\right]=1$. Similarly, in the second phase, the best relay in the decodable set forwards the signal to the CD. As such, the received signal at the CD can be mathematically written as

$$
y_{D}=\sqrt{P_{R}} h_{2 i} x_{R}+n_{i}, \quad i \in D,
$$

where $x_{R}$ denotes the signal transmitted by the selected relay and $D$ represents the decodable set of relays. Similarly, we set $E\left[\left|x_{R}\right|^{2}\right]=1$. Therefore, the signal-to-noise ratio (SNR) at $R_{i}$ and $\mathrm{CD}$ can be denoted by

$$
\begin{gathered}
\gamma_{S R_{i}}=\frac{Q\left|h_{1 i}\right|^{2}}{\max _{j}\left|h_{3 j}\right|^{2} N_{0}}, \\
\gamma_{R_{i} D}=\frac{Q\left|h_{2 i}\right|^{2}}{\max _{i \in D} \max _{j}\left|h_{i j}\right|^{2} N_{0}},
\end{gathered}
$$

respectively. In this paper, we are interested in characterizing the outage probability of the system, which is defined as the probability that the instantaneous capacity falls below a predefined rate threshold. Therefore, there are two possible cases for the data transmission depending on whether the decoding set is empty or not.

Case $1(D=\phi)$. This case corresponds that all the cognitive relays cannot decode the signal transmitted by cognitive transmitter. The probability of this case can be denoted by

$$
\operatorname{Pr}\{D=\phi\}=\operatorname{Pr}\left\{\gamma_{S R_{1}}<\gamma, \ldots, \gamma_{S R_{M}}<\gamma\right\},
$$

where $\gamma$ denotes the threshold SNR of the considered system. Due to the common variable, the probability of this case cannot be derived in the traditional method. As such, we set $X=\max _{j}\left|h_{3 j}\right|^{2}$. Therefore, the probability of this case conditioned on $X$ can be expressed as

$$
\begin{aligned}
\operatorname{Pr}\{D=\phi \mid X=x\} & =\operatorname{Pr}\left\{\gamma_{S R_{1}}<\gamma, \ldots, \gamma_{S R_{M}}<\gamma \mid X=x\right\} \\
& =\left[\operatorname{Pr}\left\{\gamma_{S R_{1}}<\gamma \mid X=x\right\}\right]^{M} \\
& =\left[\operatorname{Pr}\left\{\frac{Q\left|h_{1 i}\right|^{2}}{x N_{0}}<\gamma \mid X=x\right\}\right]^{M} \\
& =\left[\operatorname{Pr}\left\{\left|h_{1 i}\right|^{2}<\frac{N_{0} \gamma}{Q} x \mid X=x\right\}\right]^{M} \\
& =\left[\frac{\gamma\left(m_{1},\left(\beta_{1} N_{0} \gamma / Q\right) x\right)}{\Gamma\left(m_{1}\right)}\right]^{M} .
\end{aligned}
$$

According to the conclusion in [18], $\left[\gamma\left(m_{1},\left(\beta_{1} N_{0} \gamma / Q\right) x\right) /\right.$ $\left.\Gamma\left(m_{1}\right)\right]^{M}$ can be expanded as

$$
\begin{aligned}
& {\left[\frac{\gamma\left(m_{1},\left(\beta_{1} N_{0} \gamma / Q\right) x\right)}{\Gamma\left(m_{1}\right)}\right]^{M}} \\
& \quad=\frac{M \beta_{1}^{m_{1}}}{\Gamma\left(m_{1}\right)} \sum_{k=0}^{M-1}\left(\begin{array}{c}
M-1 \\
k
\end{array}\right) \\
& \quad \times(-1)^{k} e^{-\beta_{1}(1+k) x} \sum_{l=0}^{k\left(m_{1}-1\right)} \tau_{l}^{k} \beta_{1}^{l} x^{m_{1}+l-1},
\end{aligned}
$$

where $\tau_{0}^{k}=1, \tau_{1}^{k}=i, \tau_{\left(m_{1}-1\right) k}^{k}=\left(1 /\left(m_{1}-1\right) !\right)^{k}, \tau_{l}^{i}=$ $(1 / l) \sum_{v=0}^{p}((v(k+1)-l) / v !) \tau_{l-v}^{k}, p=\min \left(l, m_{1}-1\right)$, and $2 \leq$ $l \leq k\left(m_{1}-1\right)-1$. Due to the fact that $X$ is the maximum of $M$ independent nonidentical distributed Nakagami-m variables, the PDF of $X$ can be denoted by

$$
\begin{aligned}
f_{X}(x)= & \frac{N \beta_{3}^{m_{3}}}{\Gamma\left(m_{3}\right)} \sum_{m=0}^{N-1}\left(\begin{array}{c}
N-1 \\
m
\end{array}\right)(-1)^{m} \\
& \times e^{-\beta_{3}(1+m) x} \sum_{n=0}^{m\left(m_{3}-1\right)} \tau_{n}^{m} \beta_{3}^{n} x^{m_{3}+n-1} .
\end{aligned}
$$

Herein, the probability of this case can be derived as

$$
\begin{aligned}
& \operatorname{Pr}\{D=\phi\}=\int_{0}^{\infty} f_{X}(x) \operatorname{Pr}\{D=\phi \mid X=x\} d x \\
& =\int_{0}^{\infty} \frac{N \beta_{3}^{m_{3}}}{\Gamma\left(m_{3}\right)} \sum_{m=0}^{N-1}\left(\begin{array}{c}
N-1 \\
m
\end{array}\right)(-1)^{m} e^{-\beta_{3}(1+m) x} \\
& \times \sum_{n=0}^{m\left(m_{3}-1\right)} \tau_{n}^{m} \beta_{3}^{n} x^{m_{3}+n-1} \\
& \times \frac{M \beta_{1}^{m_{1}}}{\Gamma\left(m_{1}\right)} \sum_{k=0}^{M-1}\left(\begin{array}{c}
M-1 \\
k
\end{array}\right)(-1)^{k} e^{-\beta_{1}(1+k) x} \\
& \times \sum_{l=0}^{k\left(m_{1}-1\right)} \tau_{l}^{k} \beta_{1}^{l} x^{m_{1}+l-1} d x \\
& =\frac{M N \beta_{1}^{m_{1}} \beta_{3}^{m_{3}}}{\Gamma\left(m_{1}\right) \Gamma\left(m_{3}\right)} \sum_{m=0}^{N-1}\left(\begin{array}{c}
N-1 \\
m
\end{array}\right)(-1)^{m} \\
& \times \sum_{n=0}^{m\left(m_{3}-1\right)} \tau_{n}^{m} \beta_{3}^{n} \sum_{k=0}^{M-1}\left(\begin{array}{c}
M-1 \\
k
\end{array}\right)(-1)^{k} \\
& \times \sum_{l=0}^{k\left(m_{1}-1\right)} \tau_{l}^{k} \beta_{1}^{l} I_{1}
\end{aligned}
$$


where $I_{1}$ can be expressed as

$$
\begin{aligned}
I_{1} & =\int_{0}^{\infty} e^{-\left[\beta_{1}(1+k)+\beta_{3}(1+m)\right] x} x^{m_{1}+m_{3}+n+l-2} d x \\
& =\frac{\left(m_{1}+m_{3}+n+l-2\right) !}{\left[\beta_{1}(1+k)+\beta_{3}(1+m)\right]^{\left(m_{1}+m_{3}+n+l-1\right)}} .
\end{aligned}
$$

In this case, the outage probability of cognitive system can be expressed as

$$
\operatorname{Pr}\{\text { outage } \mid D=\phi\}=1 \text {. }
$$

Case $2(D=q)$. This case corresponds that $q$ cognitive relays can decode the signal transmitted by cognitive transmitter. The probability of this case can be denoted by

$$
\begin{aligned}
\operatorname{Pr}\{D=q\}= & \left(\begin{array}{c}
M \\
q
\end{array}\right) \underbrace{\operatorname{Pr}\left\{\gamma_{S R_{1}}>\gamma, \ldots, \gamma_{S R_{q}}>\gamma\right\}}_{I_{2}} \\
& \times \underbrace{\operatorname{Pr}\left\{\gamma_{S R_{q+1}}<\gamma, \ldots, \gamma_{S R_{M}}<\gamma\right\}}_{I_{3}},
\end{aligned}
$$

where $I_{2}$ is expressed as

$$
I_{2}=\operatorname{Pr}\left\{\gamma_{S R_{1}}>\gamma, \ldots, \gamma_{S R_{q}}>\gamma\right\} .
$$

Similarly, $I_{2}$ conditioned on $X$ can be mathematically written as

$$
\begin{aligned}
I_{2 \mid X=x} & =\operatorname{Pr}\left\{\gamma_{S R_{1}}>\gamma, \ldots, \gamma_{S R_{q}}>\gamma \mid X=x\right\} \\
& =\left[\operatorname{Pr}\left\{\gamma_{S R_{1}}>\gamma \mid X=x\right\}\right]^{q} \\
& =\left[1-\operatorname{Pr}\left\{\gamma_{S R_{1}}<\gamma \mid X=x\right\}\right]^{q} \\
& =\left[1-\frac{\gamma\left(m_{1},\left(\beta_{1} N_{0} \gamma / Q\right) x\right)}{\Gamma\left(m_{1}\right)}\right]^{q} .
\end{aligned}
$$

Apply the expansion for an incomplete gamma function as follows:

$$
\gamma(n, x)=\Gamma(n)\left(1-e^{-x} \sum_{i=0}^{n-1} \frac{x^{i}}{i !}\right) .
$$

We can evaluate the $I_{2 \mid X=x}$ as

$$
\begin{aligned}
I_{2 \mid X=x}= & {\left[1-\frac{\gamma\left(m_{1},\left(\beta_{1} N_{0} \gamma x / Q\right)\right)}{\Gamma\left(m_{1}\right)}\right]^{m} } \\
= & e^{-\left(\beta_{1} N_{0} \gamma x / Q\right)} \sum_{a_{1}=0}^{m_{1}-1} \frac{\left(\beta_{1} N_{0} \gamma x / Q\right)^{a_{1}}}{a_{1} !} \\
& \cdots e^{-\left(\beta_{1} N_{0} \gamma x / Q\right)} \sum_{a_{q}=0}^{m_{1}-1} \frac{\left(\beta_{1} N_{0} \gamma x / Q\right)^{a_{q}}}{a_{q} !}
\end{aligned}
$$

$$
=e^{-\left(q \beta_{1} N_{0} \gamma x / Q\right)}
$$$$
\times \sum_{a_{1}=0}^{m_{1}-1} \cdots \sum_{a_{q}=0}^{m_{1}-1} \frac{\left(\beta_{1} N_{0} \gamma\right)^{a_{1}+\cdots+a_{q}} x^{a_{1}+\cdots+a_{q}}}{a_{1} ! \cdots a_{q} ! Q^{a_{1}+\cdots+a_{q}}} .
$$

As such, the $I_{2}$ can be derived as

$$
\begin{aligned}
I_{2}= & \int_{0}^{\infty} f_{X}(x) I_{2 \mid X=x} d x \\
= & \int_{0}^{\infty} \frac{N \beta_{3}^{m_{3}}}{\Gamma\left(m_{3}\right)} \sum_{m=0}^{N-1}\left(\begin{array}{c}
N-1 \\
m
\end{array}\right)(-1)^{m} e^{-\beta_{3}(1+m) x} \\
& \times \sum_{n=0}^{m\left(m_{3}-1\right)} \tau_{n}^{m} \beta_{3}^{n} x^{m_{3}+n-1} e^{-\left(q \beta_{1} N_{0} \gamma x / Q\right)} \\
& \times \sum_{a_{1}=0}^{m_{1}-1} \cdots \sum_{a_{q}=0}^{m_{1}-1} \frac{\left(\beta_{1} N_{0} \gamma\right)^{a_{1}+\cdots+a_{q}} x^{a_{1}+\cdots+a_{q}}}{a_{1} ! \cdots a_{q} ! Q^{a_{1}+\cdots+a_{q}}} d x \\
= & \frac{N \beta_{3}^{m_{3}}}{\Gamma\left(m_{3}\right)} \sum_{m=0}^{N-1}\left(\begin{array}{c}
N-1 \\
m
\end{array}\right)(-1)^{m} \\
& \times \sum_{n=0}^{m\left(m_{3}-1\right)} \tau_{n}^{m} \beta_{3}^{n} \sum_{a_{1}=0}^{m_{1}-1} \cdots \sum_{a_{q}=0}^{m_{1}-1} \frac{\left(\beta_{1} N_{0} \gamma\right)^{a_{1}+\cdots+a_{q}}}{a_{1} ! \cdots a_{q} ! Q^{a_{1}+\cdots+a_{q}}} I_{4},
\end{aligned}
$$

where $I_{4}$ is denoted by

$$
\begin{aligned}
I_{4} & =\int_{0}^{\infty} e^{-\left[\beta_{3}(1+m)+\left(q \beta_{1} N_{0} \gamma / Q\right)\right] x} x^{m_{3}+n+a_{1}+\cdots+a_{q}-1} d x \\
& =\frac{\left(m_{3}+n+a_{1}+\cdots+a_{q}-1\right) !}{\left[\beta_{3}(1+m)+\left(q \beta_{1} N_{0} \gamma / Q\right)\right]^{\left(m_{3}+n+a_{1}+\cdots+a_{q}\right)}} .
\end{aligned}
$$

The exact of $I_{3}$ can be easily deduced by substituting the parameters with their respective counterparts; that is, $M \rightarrow$ $M-m$. In this case, the outage probability of cognitive system can be expressed as

$$
\begin{aligned}
& \operatorname{Pr}\{\text { outage } \mid D=q\} \\
& =\operatorname{Pr}\left\{\max _{i \in D}\left\{\frac{Q\left|h_{2 i}\right|^{2}}{\max _{j}\left|h_{i j}\right|^{2} N_{0}}\right\}<\gamma \mid D=q\right\} \\
& =[\underbrace{\operatorname{Pr}\left\{\frac{Q\left|h_{2 i}\right|^{2}}{\max _{j}\left|h_{i j}\right|^{2} N_{0}}<\gamma\right\}}_{I_{5}}]^{q} .
\end{aligned}
$$


As such, the $I_{5}$ can be deduced as

$$
\begin{aligned}
& I_{5}=\int_{0}^{\infty} f_{\max _{j}\left|h_{i j}\right|^{2}}(y) \int_{0}^{N_{0} \gamma y / Q} f_{\left|h_{1}\right|^{2}}(z) d z d y \\
& =\int_{0}^{\infty} f_{\max _{j}\left|h_{i j}\right|^{2}}(y) f_{\left|h_{1}\right|^{2}}\left(\frac{N_{0} \gamma y}{Q}\right) d y \\
& =\int_{0}^{\infty} \frac{N \beta_{4}^{m_{4}}}{\Gamma\left(m_{4}\right)} \sum_{r=0}^{N-1}\left(\begin{array}{c}
N-1 \\
r
\end{array}\right)(-1)^{r} e^{-\beta_{4}(1+r) y} \\
& \times \sum_{s=0}^{r\left(m_{4}-1\right)} \tau_{s}^{r} \beta_{4}^{s} y^{m_{4}+s-1} \frac{\gamma\left(m_{2},\left(\beta_{2} N_{0} \gamma y / Q\right)\right)}{\Gamma\left(m_{2}\right)} d y \\
& =1-\int_{0}^{\infty} \frac{N \beta_{4}^{m_{4}}}{\Gamma\left(m_{4}\right)} \sum_{r=0}^{N-1}\left(\begin{array}{c}
N-1 \\
r
\end{array}\right)(-1)^{r} e^{-\beta_{4}(1+r) y} \\
& \times \sum_{s=0}^{r\left(m_{4}-1\right)} \tau_{s}^{r} \beta_{4}^{s} y^{m_{4}+s-1} e^{-\left(\beta_{2} N_{0} \gamma / Q\right) y} \\
& \times \sum_{t=0}^{m_{2}-1} \frac{\left(\beta_{2} N_{0} \gamma y / Q\right)^{t}}{t !} d y \\
& =1-\frac{N \beta_{4}^{m_{4}}}{\Gamma\left(m_{4}\right)} \sum_{r=0}^{N-1}\left(\begin{array}{c}
N-1 \\
r
\end{array}\right)(-1)^{r} \\
& \times \sum_{s=0}^{r\left(m_{4}-1\right)} \tau_{s}^{r} \beta_{4}^{s} \sum_{t=0}^{m_{2}-1} \frac{\left(\beta_{2} N_{0} \gamma / Q\right)^{t}}{t !} I_{6},
\end{aligned}
$$

where $I_{6}$ can be expressed as

$$
\begin{aligned}
I_{6}= & \int_{0}^{\infty} y^{m_{4}+s+t-1} e^{-\left[\beta_{4}(1+r)+\left(\beta_{2} N_{0} \gamma / Q\right)\right] y} d y \\
= & \left(m_{4}+s+t-1\right) ! \\
& \times\left[\beta_{4}(1+r)+\frac{\beta_{2} N_{0} \gamma}{Q}\right]^{-\left(m_{4}+s+t\right)} .
\end{aligned}
$$

To this end, the outage probability of the considered system can be derived as

$$
\begin{aligned}
P_{\text {out }}= & \operatorname{Pr}\{D=\phi\} \operatorname{Pr}\{\text { outage } \mid D=\phi\} \\
& +\sum_{q=1}^{M} \operatorname{Pr}\{D=q\} \operatorname{Pr}\{\text { outage } \mid D=q\},
\end{aligned}
$$

where $\operatorname{Pr}\{D=\phi\}, \operatorname{Pr}$ \{outage $\mid D=\phi\}, \operatorname{Pr}\{D=m\}$, and Pr\{outage $\mid D=m\}$ are calculated as (13), (15), (16), and (23), respectively. Thus, the closed form of outage probability has been derived.

\section{Numerical Results}

In this section, we numerically evaluate the outage probability of the considered system. Specifically, all simulation results are obtained by taking expectation over $10^{8}$ independent trials.

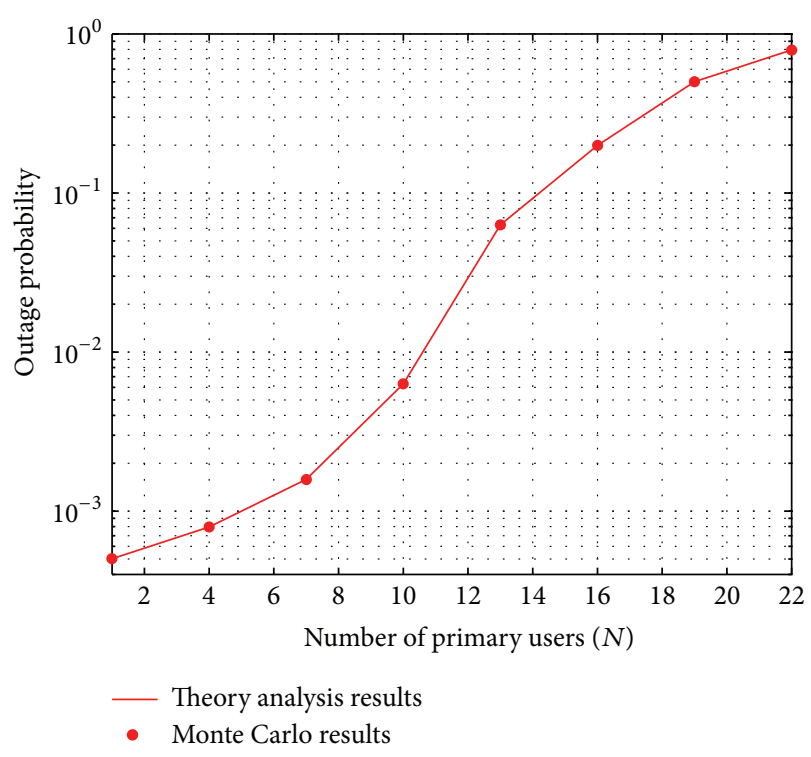

FIGURE 2: OP of CRN: varying the number of PUs when $Q=10 \mathrm{~dB}$.

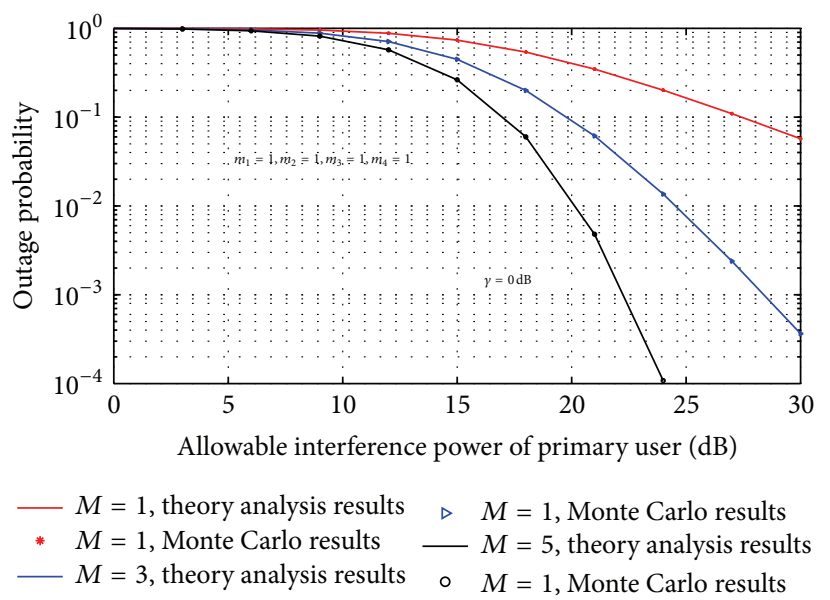

FIGURE 3: OP of CRN: varying the maximum allowable interference power of PU when $m_{1}=1, m_{2}=1, m_{3}=1$, and $m_{4}=1$.

Firstly, the outage performance of cognitive system versus number of primary users is presented in Figure 2. From Figure 2, the outage performance will deteriorate when the number of primary users increases. Moreover, the Monte Carlo results match perfectly with the theory analysis results, which validates the theory analysis.

Secondly, we evaluate the outage performance of cognitive system under adjustable maximum interference power of primary users with different number of relays. Results which are shown in Figure 3 illustrate that the outage performance will improve when the maximum allowable interference power of primary users increases. Specifically, the number of cognitive relays affects the diversity of the considered system. Similarly, the Monte Carlo results match perfectly with the theory analysis results.

Thirdly, the outage performance of cognitive system versus number of cognitive relays is depicted in Figure 4. We 


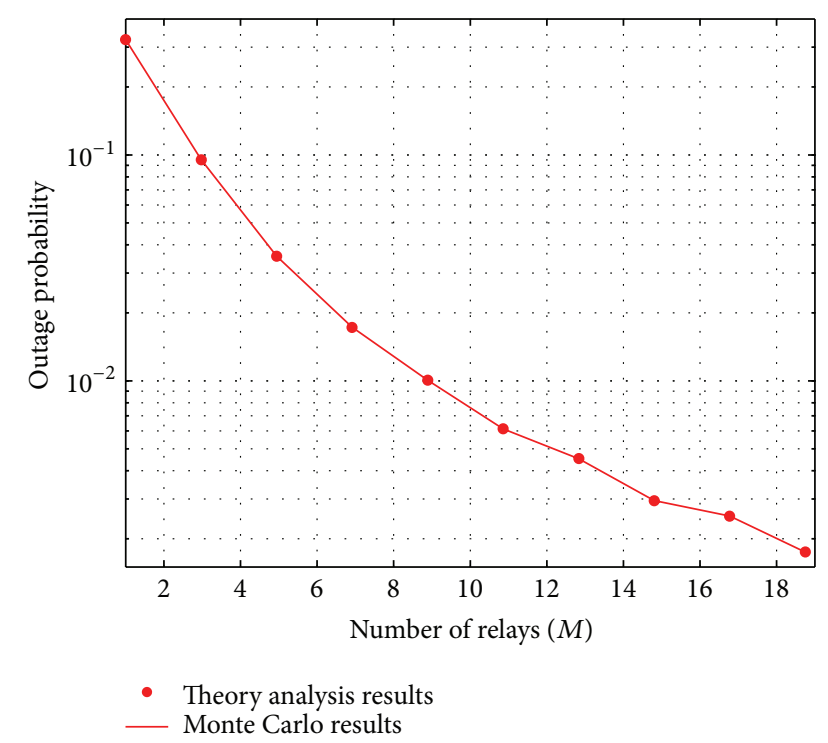

FIGURE 4: OP of CRN: varying the number of cognitive relays when $N=3$.

can directly see from Figure 4 that the outage performance will improve greatly when the number of cognitive relays increases.

Lastly, we evaluate the outage performance of cognitive system under adjustable channel gains. We can directly see from Figure 5 that the outage performance will improve when the channel quality of cognitive system increases or when the channel between cognitive system and primary system is in deep fading. Specifically, channel gains are not related to the diversity of the considered system. Based on simulation results, the obtained analytical results can guide us to design the system to maintain the outage probability.

\section{Conclusions}

In this paper, incorporating the best relay selection in a decode-and-forward cooperative spectrum sharing system subject to independent nonidentical Nakagami-m fading channels, the closed-form expression of outage probability was derived. Based on closed-form expression, we can evaluate the impacts of maximum allowable interference power of primary users, number of primary users, number of cognitive relays, and channel gains on the outage performance of cognitive system. Specifically, we validated our theory analysis through Monte Carlo simulation and found that the diversity is affected by number of cognitive relays.

\section{Conflict of Interests}

The authors declare that there is no conflict of interests regarding the publication of this paper.

\section{Acknowledgments}

This work was supported by the National Basic Research Program (973) of China under Grant no. 2009CB320400,

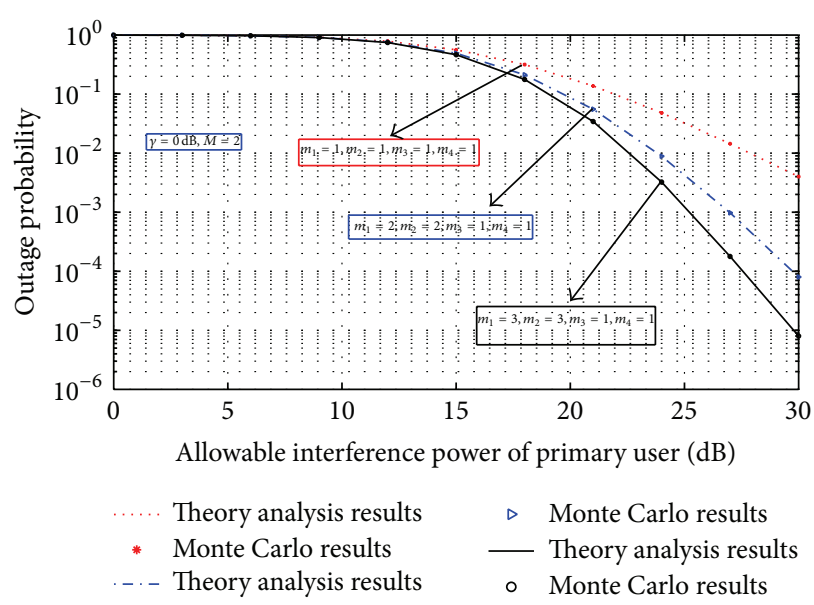

(a)

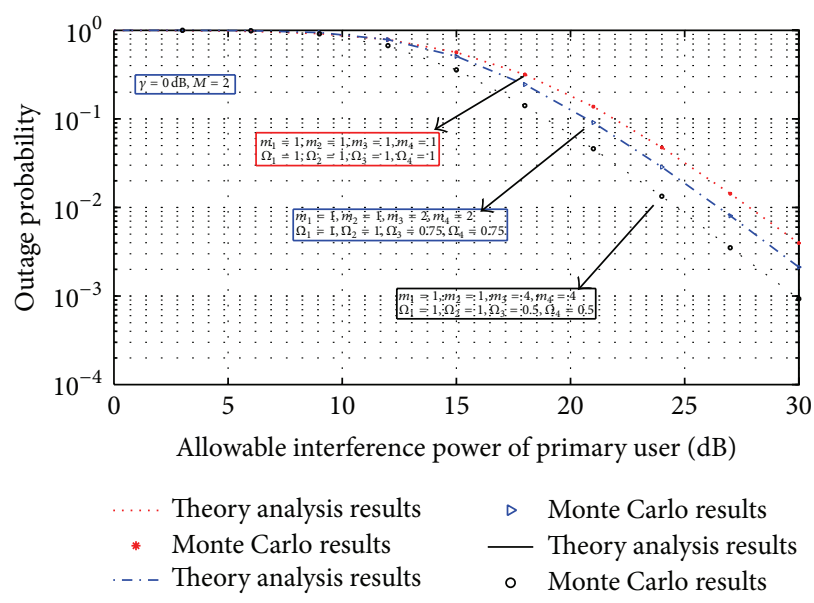

(b)

FIGURE 5: OP of CRN: varying the channel gain when $N=$ 3 and $M=3 \mathrm{~dB}$.

by the National Science Foundation of China under Grant no. 60932002 and no. 61172062, and in part by the Jiangsu Province Natural Science Foundation of China under Grant no. BK2011116.

\section{References}

[1] Federal Communications Commission (FCC), ET Docket No. 03-322 Notice of Proposed Rule Making and Order, 2003.

[2] S. Haykin, "Cognitive radio: brain-empowered wireless communications," IEEE Journal on Selected Areas in Communications, vol. 23, no. 2, pp. 201-220, 2005.

[3] Q. Wu, H. Han, J. Wang, Z. Zhao, and Z. Zhang, "Sensing task allocation for heterogeneous channels in cooperative spectrum sensing," Radioengineering, vol. 19, no. 4, pp. 544-551, 2010.

[4] Y. Xu, J. Wang, Q. Wu, Z. Zhang, A. Anpalagan, and L. Shen, "Optimal energy-efficient channel exploration for opportunistic spectrum usage," IEEE Wireless Commmunication Letter, vol. 1, no. 2, pp. 77-80, 2012. 
[5] Z. Zhang, Q. Wu, and J. Wang, "Energy-efficient power allocation strategy in cognitive relay networks," Radioengineering, vol. 21, no. 3, pp. 809-814, 2012.

[6] J. N. Laneman, D. N. C. Tse, and G. W. Wornell, "Cooperative diversity in wireless networks: efficient protocols and outage behavior," IEEE Transactions on Information Theory, vol. 50, no. 12, pp. 3062-3080, 2004.

[7] T. E. Hunter, S. Sanayei, and A. Nosratinia, "Outage analysis of coded cooperation," IEEE Transactions on Information Theory, vol. 52, no. 2, pp. 375-391, 2006.

[8] Y. Zou, B. Zheng, and J. Zhu, "Outage analysis of opportunistic cooperation over rayleigh fading channels," IEEE Transactions on Wireless Communications, vol. 8, no. 6, pp. 3077-3085, 2009.

[9] Y.Zou, B. Zheng, and W.-P. Zhu, "An opportunistic cooperation scheme and its BER analysis," IEEE Transactions on Wireless Communications, vol. 8, no. 9, pp. 4492-4497, 2009.

[10] S. Sagong, J. Lee, and D. Hong, "Capacity of reactive DF scheme in cognitive relay networks," IEEE Transactions on Wireless Communications, vol. 10, no. 10, pp. 3133-3138, 2011.

[11] Y. Guo, G. Kang, N. Zhang, W. Zhou, and P. Zhang, "Outage performance of relay-assisted cognitive-radio system under spectrum-sharing constraints," Electronics Letters, vol. 46, no. 2, pp. 182-184, 2010.

[12] L. Luo, P. Zhang, G. Zhang, and J. Qin, "Outage performance for cognitive relay networks with underlay spectrum sharing," IEEE Communications Letters, vol. 15, no. 7, pp. 710-712, 2011.

[13] J. Lee, H. Wang, J. G. Andrews, and D. Hong, "Outage probability of cognitive relay networks with interference constraints," IEEE Transactions on Wireless Communications, vol. 10, no. 2, pp. 390-395, 2011.

[14] T. Q. Duong, P. L. Yeoh, V. N. Q. Bao, M. Elkashlan, and N. Yang, "Cognitive relay networks with multiple primary transceivers under spectrum-sharing," IEEE Signal Processing Letters, vol. 19, no. 11, pp. 741-744, 2012.

[15] T. Q. Duong, D. Benevides da Costa, M. Elkashlan, and V. N. Quoc Bao, "Cognitive amplify-and-forward relay networks over Nakagami-m fading," IEEE Transactions on Vehicular Technology, vol. 61, no. 5, pp. 2368-22374, 2012.

[16] C. Zhong, T. Ratnarajah, and K.-K. Wong, "Outage analysis of decode-and-forward cognitive dual-hop systems with the interference constraint in Nakagami-m fading channels," IEEE Transactions on Vehicular Technology, vol. 60, no. 6, pp. 28752879, 2011.

[17] T. Q. Duong et al., "Outage and diversity of cognitive relaying systems under spectrum sharing in Nakagami-m fading," IEEE Communications Letters, vol. 16, no. 12, pp. 2075-2078, 2012.

[18] D. B. da Costa, M. Elkashlan, P. Lep Yeoh, N. Yang, and M. D. Yacoub, "Dual-hop cooperative spectrum sharing systems with multi-primary users and multi-secondary destinations over Nakagami-m fading," in Proceedings of the IEEE 23rd International Symposium on Personal, Indoor and Mobile Radio Communications (PIMRC '12), 2012.

[19] I. S. Gradshteyn and I. M. Ryzhik, Table of Integrals, Series, and Products, Academic Press, Orlando, Fla, USA, 5th edition, 1994. 


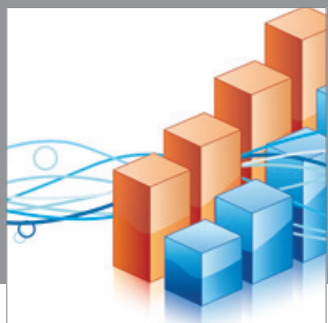

Advances in

Operations Research

mansans

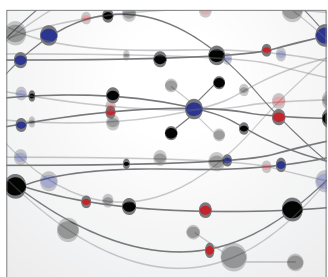

The Scientific World Journal
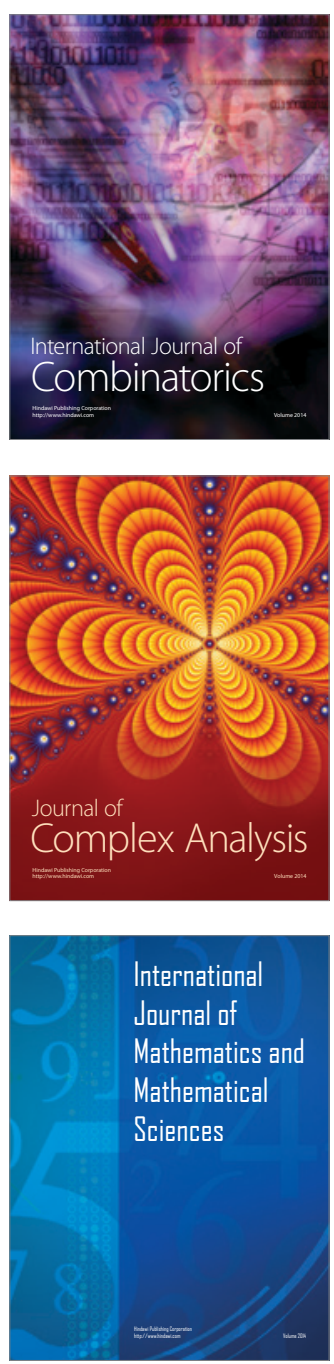
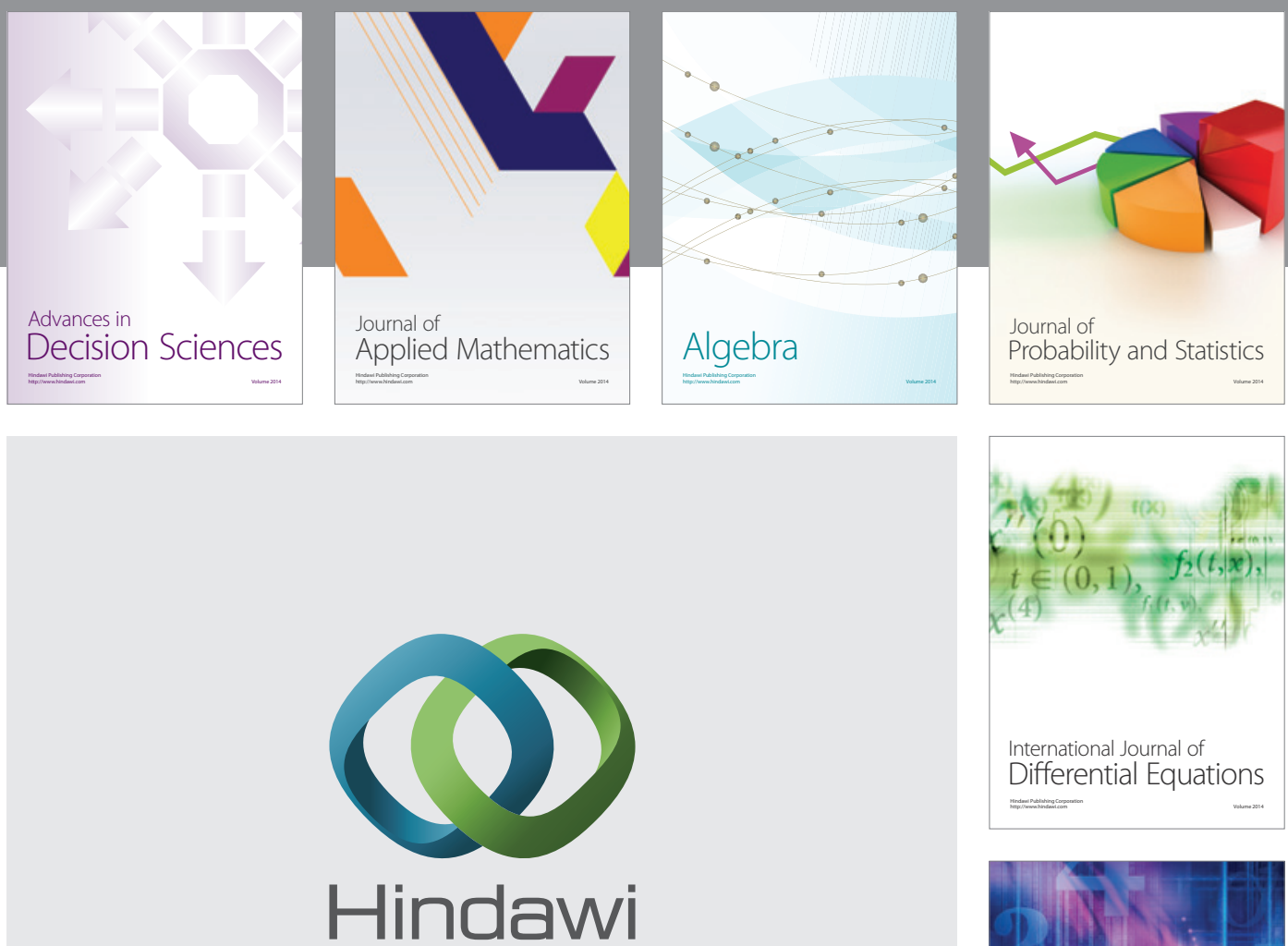

Submit your manuscripts at http://www.hindawi.com
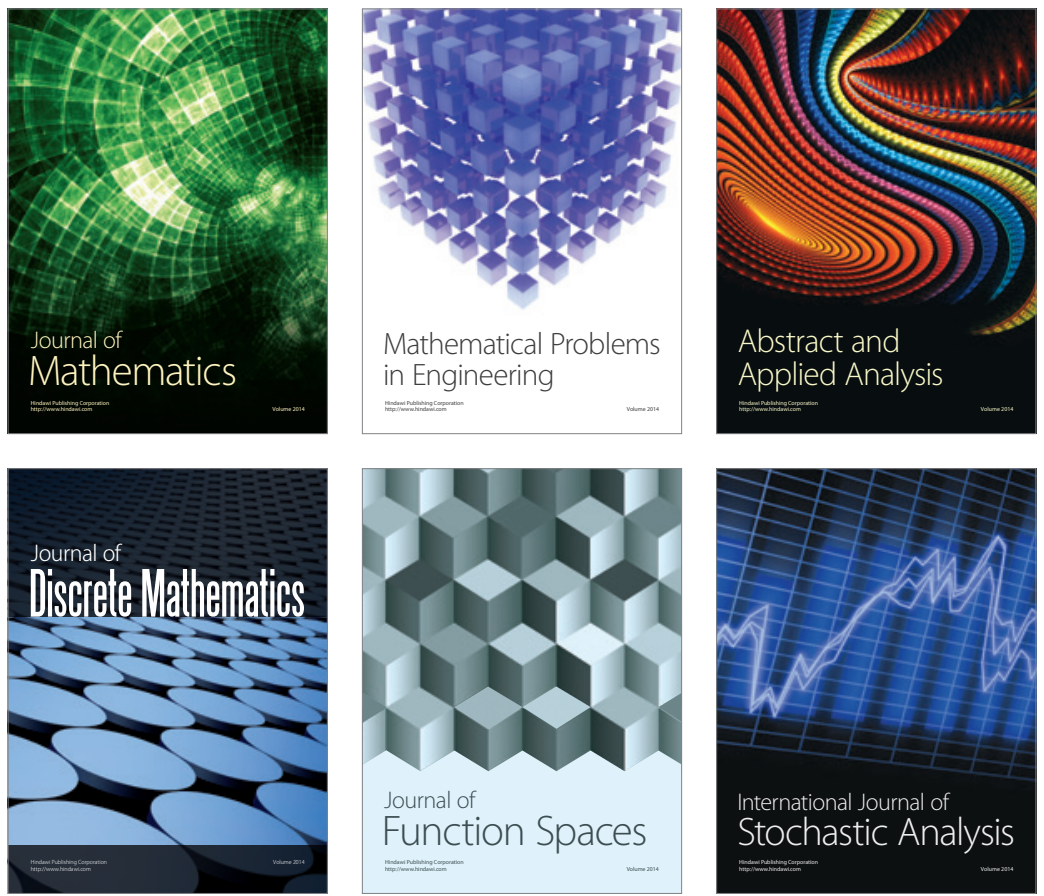

Journal of

Function Spaces

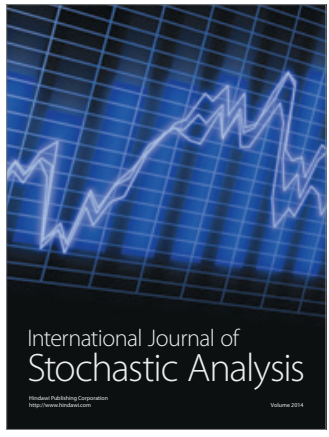

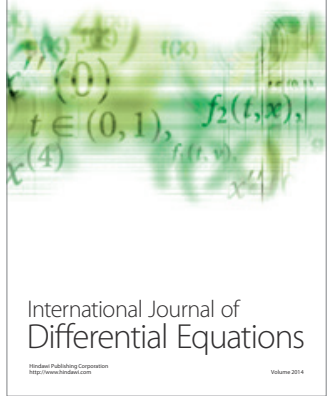
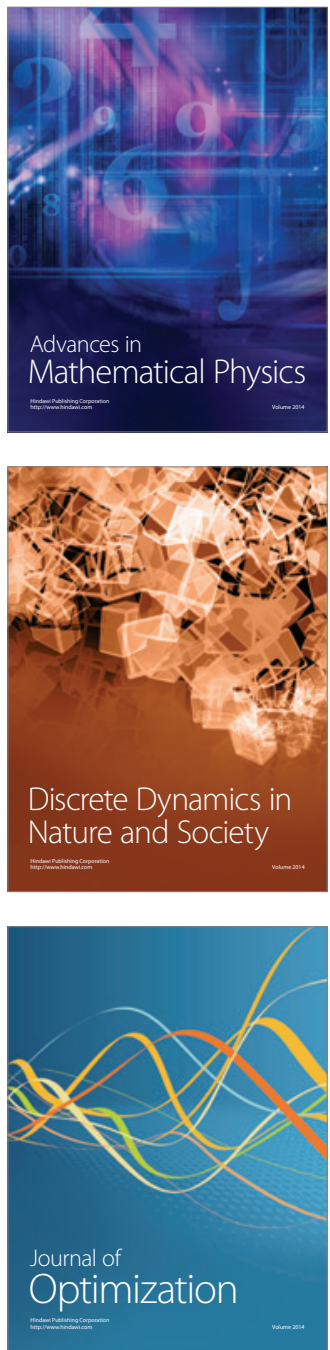